\section{Fatores associados ao uso de benzodiazepínicos no serviço municipal de saúde da cidade de Coronel Fabriciano, Minas Gerais, Brasil}

\author{
Factors associated with benzodiazepine prescription \\ by local health services in Coronel Fabriciano, \\ Minas Gerais State, Brazil
}

\author{
${ }^{1}$ Faculdade de Farmácia, \\ Universidade Federal de \\ Minas Gerais, Belo Horizonte, \\ Brasil. \\ 2 Faculdade de Odontologia, \\ Universidade Federal de \\ Minas Gerais, Belo Horizonte, \\ Brasil. \\ Correspondência \\ M. H. N. G. Abreu \\ Faculdade de Odontologia \\ Universidade Federal de \\ Minas Gerais. \\ Rua Arthur Ferrari 55, apto. \\ 401, Belo Horizonte, $M G$ \\ 30431-210, Brasil \\ maurohenriqueabreu@ig.com.br
}

\section{Abstract}

Numerous countries have witnessed increasing misuse of benzodiazepines. This cross-sectional study assessed the factors associated with prescription of benzodiazepines for users of the Municipal Health Service in Colonel Fabriciano, Minas Gerais State, Brazil. Data were collected from official records on drug prescription $(n=1,866)$ from September to October 2006. Bivariate and multivariate analyses using Poisson regression were performed. Prolonged benzodiazepine use was independently associated with benzodiazepine type and patient participation in health programs. Concomitant use of other psychoactive drugs was independently associated with age and participation in health programs. Type of benzodiazepine (clonazepam or diazepam) was independently associated with age and gender, as well as with participation in health programs. Factors associated with benzodiazepine prescription show the problem's extent and should be considered in planning interventions to rationalize the use of these drugs in Brazil, particularly through health program planning.

Benzodiazepines; Psychotropic Drugs; Pharmacoepidemiology
Karleyla Fassarela Firmino 1

Mauro Henrique Nogueira Guimarães de Abreu 2

Edson Perini 1

Sérgia Maria Starling Magalhães 1

\section{Introdução}

A partir da segunda metade do século XX, iniciou-se na medicina uma revolução tecnológica, incorporando recursos diagnósticos e terapêuticos que mudaram a abordagem das doenças. A psiquiatria tradicional ganhou com o benzodiazepínico um inestimável aliado, criando expectativa de resolutividade segura para alguns quadros como a ansiedade e a insônia ${ }^{1}$.

Os benzodiazepínicos, introduzidos na terapêutica na década de 1960, são fármacos depressores do sistema nervoso central (SNC), utilizados como hipnóticos, ansiolíticos, anticonvulsivantes e miorrelaxantes 2 . A eficácia dos benzodiazepínicos é bem documentada nos tratamentos de curta duração, porém o uso prolongado é contraindicado devido aos riscos de efeitos adversos, incluindo a dependência. Ao longo do tempo, com a popularização do uso dos benzodiazepínicos novos problemas foram evidenciados, grande parte deles, decorrentes do mau uso desses medicamentos. A dependência química dos benzodiazepínicos com todas as implicações inerentes a esses quadros passaram a constituir grande preocupação para a saúde pública 3 .

Diversos estudos 4,5,6,7,8,9,10 já apontaram distorções nas prescrições de benzodiazepínicos, como o tempo de tratamento prolongado, uso equivocado para o alívio de quadros inespecíficos, uso por idosos e outras indicações incompatíveis com o perfil farmacológico da classe. Os 
múltiplos agravos à saúde causados por esses medicamentos podem ainda ser potencializados pelo uso concomitante de outros depressores do SNC como álcool e outros psicotrópicos 2 .

As questões relacionadas ao uso excessivo e por vezes injustificado dos benzodiazepínicos são observadas em diversos países, independente do seu grau de desenvolvimento econômico $4,5,6,7,8,9,10$, nos grandes centros urbanos 6,9 e também nas populações rurais 11. No Brasil, estimase que $1,6 \%$ da população adulta seja usuária crônica de benzodiazepínicos 12. Em grandes cidades brasileiras estudos confirmam o uso indiscriminado dessa classe de medicamentos 9,13. Em Coronel Fabriciano, cidade do interior de Minas Gerais, Brasil, o problema se mostra igualmente importante.

No Serviço Público de Saúde de Coronel Fabriciano tem sido observado um elevado consumo dos dois benzodiazepínicos padronizados no município - Diazepam e Clonazepam. Esse uso exacerbado vem suscitando discussões entre os profissionais envolvidos com o problema na região acerca dos fatores associados à utilização desses medicamentos, levando-se em consideração as evidências de que características culturais locais, a disponibilidade de medicamentos, as variações nos padrões nosológicos e o acesso aos serviços de saúde podem ser fatores determinantes do comportamento diferenciado entre as localidades.

Nesse contexto, a proposta desse estudo foi avaliar os fatores associados à terapia por tempo prolongado com benzodiazepínicos, ao uso concomitante de benzodiazepínicos e outros psicotrópicos e a prescrição do Diazepam.

\section{Materiais e métodos}

O Município de Coronel Fabriciano, localizado na região leste do Estado de Minas Gerais, apresenta população de 102.588 habitantes. Conhecido como uma "cidade dormitório", suas atividades econômicas estão centradas na atividade industrial e no comércio e grande parte da população trabalha nas atividades ligadas às siderúrgicas dos municípios vizinhos.

O serviço municipal de saúde é composto por 10 unidades básicas de saúde (UBS) que conta com clínicos gerais, ginecologistas e pediatras, um centro de especialidades (CEPS) responsável pela atenção secundária com atendimento das especialidades médicas: cardiologia, oftalmologia, dermatologia, urologia, otorrinolaringologia, pneumologia e mastologia, duas equipes do Programa Saúde da Família (PSF), um Centro de Atenção a Saúde Mental (CASAM) com atendi- mento de neurologia e psiquiatria e um hospital conveniado.

A dispensação de medicamentos é feita nas farmácias das UBS, com exceção dos medicamentos psicotrópicos, dispensados na Farmácia Central. Em 2002 foi implantado um sistema informatizado para a dispensação de medicamentos. No momento desse estudo o sistema possuía cerca de 58 mil pacientes cadastrados no Serviço Municipal de Saúde. A lista de medicamentos para a atenção básica do município em 2006 contava com 173 itens, incluindo o Diazepam 10mg, Clonazepam 2mg e Clonazepam 2,5mg/mL, dispensados mediante notificação de receita $\mathrm{B}$, de acordo com a legislação que aprova o regulamento técnico sobre substâncias e medicamentos sujeitos a controle especial - Portaria $n^{o}$. 344/98 da Secretaria de Vigilância em Saúde, Ministério da Saúde em 12 de maio de $1998{ }^{14}$.

Trata-se de um estudo transversal com coleta de dados dos meses de setembro e outubro de 2006. Foi realizada a avaliação do total das notificações de receita de benzodiazepínicos originadas no Serviço Municipal de Saúde e retidas na Farmácia Central. A escolha do período teve por objetivo evitar receitas de retorno do paciente ao serviço, pois a legislação estabelece a dispensação dos psicotrópicos para um período máximo de dois meses de tratamento 14 .

Os dados foram coletados a partir das notificações de receita e do cadastro do paciente no serviço. Foram incluídas no estudo as notificações de receitas provenientes das unidades de atenção primária e secundária, devidamente preenchidas, contendo o número de cadastro do paciente. Se a notificação de receita não apresentava o número de cadastro do paciente, buscava-se localizá-lo pelo nome e, se este estivesse incompleto ou parcialmente ilegível, verificavam-se todos os cadastros com mesmo nome para identificar qual paciente recebera prescrição naquela data, se apenas um paciente tivesse recebido, este era incluído na amostra. Foram excluídas as receitas de pacientes que não puderam ser identificados e a segunda prescrição daqueles que retornaram a Farmácia Central durante o período do estudo, uma vez que o desenho do estudo é transversal e a unidade de análise foi a primeira prescrição medicamentosa do paciente no período da pesquisa. Foram também excluídos os casos de preenchimento incorreto da notificação de receita. Ressalta-se que não foram identificadas infrações sanitárias que justificassem encaminhamento à vigilância sanitária do município.

A coleta seguiu um protocolo estruturado contendo dados da notificação de receita (fármaco, concentração, posologia, duração do 
tratamento) e dados sociodemográficos e de utilização do serviço, acessado no sistema informatizado. Foram coletados os seguintes dados: tipo de benzodiazepínico utilizado (Diazepam 10mg, Clonazepam 2mg e Clonazepam 2,5mg/ $\mathrm{mL}$ ); gênero do usuário; idade em anos; inserção no mercado de trabalho, duração de tratamento contínuo com o benzodiazepínicos (meses), cadastro em programas municipais de saúde de hipertensão e diabetes; uso de outros medicamentos psicotrópicos juntamente com o benzodiazepínico. A classificação dos psicotrópicos prescritos foi realizada de acordo com o sistema ATC (Anatomical Therapeutic Chemical Classification System).

O tempo de tratamento contínuo com benzodiazepínicos foi estratificado em superior a 6 meses ou até 6 meses, sendo o primeiro caso considerado como tempo de tratamento prolongado de acordo com o preconizado pela literatura 15,16. Para avaliação da inserção no mercado de trabalho usuários que se declararam aposentados e pensionistas foram classificados como sem inserção profissional.

A análise estatística envolveu, além da análise descritiva, análises bivariadas e multivariadas. As três variáveis respostas foram a prescrição de Diazepam, o tempo prolongado de tratamento e o uso concomitante do benzodiazepínico com outros psicotrópicos. As variáveis explicativas foram gênero, idade, inserção no mercado de trabalho e cadastro nos programas municipais de saúde. Para a análise bivariada dos dados, empregou-se o teste do qui-quadrado de Pearson e o teste t de Student. Modelos de regressão de Poisson foram desenvolvidos 17. Variáveis que se mantiveram associadas $(\mathrm{p}<0,20)$ na análise bivariada, foram incluídas no modelo multivariado. Foram estimadas as razões de prevalência (RP; intervalo de 95\% de confiança-IC95\%) ajustadas no modelo multivariado de cada uma das variáveis resposta. O programa empregado nas análises estatísticas foi o SPSS versão 17.0 (SPSS Inc., Chicago, Estados Unidos).

A pesquisa foi aprovada pelo Comitê de Ética em Pesquisa da Universidade Federal de Minas Gerais (COEP/UFMG; parecer no. 197/06), de acordo com a Resolução $n^{\circ}$. 196/96 do Conselho Nacional de Saúde. Por se tratar de pesquisa baseada em dados secundários, a autorização de acesso foi dada pelo secretário Municipal de Saúde. Problemas detectados no preenchimento não foram notificados à vigilância sanitária em respeito ao compromisso de sigilo assumido com a autoridade responsável pela autorização do acesso aos dados.

\section{Resultados}

Foram aviadas 2.113 receitas na Farmácia Central no período do estudo. Foram excluídas 247 $(11,69 \%)$, sendo $92(4,35 \%)$ referentes à segunda prescrição de pacientes que retornaram a Farmácia Central no período do estudo e 155 por dificuldade de identificação do cadastro do paciente. Um total de 1.866 receitas foi incluído na análise. O Diazepam 10mg foi prescrito em 59,7\% das notificações e o Clonazepam $2 \mathrm{mg}$ em 40,2\%. Apenas uma prescrição foi referente ao Clonazepam 2,5mg/mL e foi excluída da amostra.

Aproximadamente $75 \%$ das prescrições destinavam-se a mulheres e indivíduos adultos, porém o número de idosos recebendo prescrição de benzodiazepínicos foi de aproximadamente $25 \%$. A média de idade foi 49,7 anos. A distribuição dos usuários de benzodiazepínicos segundo o gênero e faixa etária é apresentada na Tabela 1.

Dos pacientes cadastrados nos programas coletivos de hipertensão e diabetes, 30,8\% eram hipertensos, $1,3 \%$ diabéticos e $4,8 \%$ hipertensos e diabéticos.

Para quase a metade dos pacientes não foi possível obter-se o dado autodeclarado de ocupação profissional remunerada. Mais de 50\% dos pacientes utilizaram algum outro psicotrópico concomitante ao benzodiazepínico (Tabela 1).

A análise bivariada foi efetuada com o objetivo de identificar fatores associados ao tempo de tratamento, a prescrição do Diazepam e ao uso do benzodiazepínico concomitante com outros psicotrópicos. Na Tabela 2 são apresentados os resultados da análise bivariada entre o tempo de tratamento e as covariáveis.

O uso prolongado de benzodiazepínico teve prevalência maior entre os usuários do Diazepam quando comparado ao Clonazepam, entre os pacientes cadastrados nos programas coletivos de saúde e entre os indivíduos com 60 anos ou mais.

Buscando-se um modelo estatístico que apontasse os principais determinantes do uso prolongado do benzodiazepínico, foram incluídas no modelo multivariado as variáveis que se mantiveram associadas na análise bivariada com $\mathrm{p}<0,20$. Na Tabela 2 são apresentados os resultados finais da análise multivariada. Os resultados revelaram que o uso de Diazepam e o cadastro nos programas de saúde apresentaram associações independentes com o tempo prolongado de tratamento com benzodiazepínicos. Deste modo, indivíduos que utilizavam Diazepam apresentaram uma frequência de uso prolongado de benzodiazepínicos 1,41 vez maior (IC95\%; 1,29$1,54)$ do que quem utilizava Clonazepam. Participantes dos programas municipais de saúde 
Tabela 1

Distribuição de frequência das variáveis gênero, faixa etária, cadastro em programas municipais de saúde e uso de outros psicotrópicos de usuários de benzodiazepínicos. Serviço Municipal de Saúde, Coronel Fabriciano, Minas Gerais, Brasil, 2006.

\begin{tabular}{lcc}
\hline Variável & $\mathbf{n}$ & \% amostra \\
\hline Gênero & & \\
$\quad$ Feminino & 1.387 & 74,3 \\
$\quad$ Masculino & 479 & 25,7 \\
Faixa etária (anos) & 1.416 & 75,9 \\
$\quad$ Menos de 60 * & 450 & 24,1 \\
60 ou mais & & \\
Inserção profissional & 666 & 35,7 \\
$\quad$ Sem inserção no mercado de trabalho & 379 & 20,3 \\
Com inserção no mercado de trabalho & 821 & 44,0 \\
$\quad$ Dado não disponível & & 36,9 \\
Cadastro em programas de saúde & 689 & 63,1 \\
Pacientes cadastros nos programas municipais de saúde & 1.177 & \\
Não cadastrado nos programas & & 44,4 \\
Uso de outros psicotrópicos & 829 & 55,6 \\
$\quad$ Uso apenas do benzodiazepínico & 1.037 & \\
Uso do benzodiazepínico associado a outros psicotrópicos & &
\end{tabular}

* Quatro pacientes tinham idade inferior a 16 anos.

Tabela 2

Associação entre o tempo prolongado de tratamento com benzodiazepínico e as variáveis explicativas. Coronel Fabriciano, Minas Gerais, Brasil, 2006.

\begin{tabular}{|c|c|c|c|c|c|}
\hline \multirow[t]{3}{*}{ Covariáveis } & \multirow{2}{*}{\multicolumn{2}{|c|}{$\begin{array}{l}\text { Tempo prolongado de tratamento com } \\
\text { benzodiazepínicos (meses) * }\end{array}$}} & \multirow{3}{*}{$\begin{array}{l}\text { Análise bivariada } \\
\text { Valor de } p * \star\end{array}$} & \multicolumn{2}{|c|}{ Análise multivariada } \\
\hline & & & & \multirow[t]{2}{*}{ RP (IC95\%) } & \multirow[t]{2}{*}{ Valor de $p$} \\
\hline & Até 6 & $>6$ & & & \\
\hline \multicolumn{6}{|l|}{ Faixa etária (anos) } \\
\hline Menos de 60 & 630 & 784 & 0,046 & & \\
\hline 60 anos ou mais & 176 & 273 & & & \\
\hline \multicolumn{6}{|l|}{ Gênero } \\
\hline Masculino & 204 & 274 & 0,764 & & \\
\hline Feminino & 602 & 783 & & & \\
\hline \multicolumn{6}{|l|}{ Inserção profissional } \\
\hline Sem inserção & 243 & 422 & 0,220 & & \\
\hline Com inserção & 153 & 226 & & & \\
\hline \multicolumn{6}{|c|}{ Medicamento prescrito } \\
\hline Diazepam & 392 & 720 & $<0,001$ & $1,41(1,29-1,54)$ & $<0,001$ \\
\hline Clonazepam & 414 & 337 & & 1,00 & \\
\hline \multicolumn{6}{|c|}{ Cadastro em programas de saúde } \\
\hline Cadastrado & 242 & 446 & $<0,001$ & $1,19(1,10-1,29)$ & $<0,001$ \\
\hline Sem cadastro & 564 & 611 & & 1,00 & \\
\hline
\end{tabular}

IC95\%: intervalo de $95 \%$ de confiança.

* Em três prescrições a variável tempo de tratamento não foi acessada;

** Teste qui-quadrado de Pearson. 
apresentaram uma frequência de uso prolongado de benzodiazepínicos 1,19 (IC95\%: 1,10-1,29) vez maior do que aqueles que não participavam dos programas.

Procurou-se estabelecer possíveis associações entre o uso de outros medicamentos psicotrópicos concomitante ao benzodiazepínico e as covariáveis sociodemográficas, de utilização de serviço e o benzodiazepínico usado (Tabela 3). Verificou-se a associação entre as variáveis idade ( $p<0,001)$, inserção profissional $(p=0,001)$, tipo de benzodiazepínicos utilizado $(\mathrm{p}<0,001)$ e cadastro em programa de saúde $(\mathrm{p}<0,001)$.

$\mathrm{Na}$ análise multivariada permaneceram como variáveis independentemente associadas ao uso concomitante de benzodiazepínicos e outros psicotrópicos a idade e o cadastro em programas de saúde. Os indivíduos adultos, com idade menor que 60 anos, têm prevalência de uso de outros psicotrópicos concomitantemente ao benzodiazepínico 1,63 (IC95\%: 1,42-1,86) vez maior do que os idosos ( $\geq 60$ anos). Pacientes que não participavam dos programas municipais de saúde tiveram uma prevalência de uso de outros psicotrópicos além do benzodiazepínico 1,18 (IC95\%: 1,01-1,30) vez maior do que aqueles que participam dos programas (Tabela 3 ).

Devido ao fato de os dois benzodiazepínicos que compõem a lista de medicamentos para a atenção básica do município terem um perfil farmacológico ligeiramente diferenciado, buscou-se avaliar as associações entre o benzodiazepínico usado (Diazepam ou Clonazepam) e as covariáveis. Os resultados da análise bivariada e multivariada são apresentados na Tabela 4 .

Os resultados indicam que indivíduos com idade de 60 anos ou mais apresentam uma prevalência de consumo de Diazepam 1,40 (IC95\%: $1,19-1,65)$ vez maior do que os mais jovens. Homens apresentaram 1,23 vez (IC95\%: 1,07-1,41) maior prevalência de consumo de Diazepam do que as mulheres. Verificou-se ainda que o cadastro em programas aumenta em 1,35 (IC95\%: 1,051,13 ) vez a prevalência de consumo de Diazepam comparado a pacientes não cadastrados.

\section{Discussão}

Nesse estudo, verificou-se a prevalência da prescrição do Diazepam, um benzodiazepínico de meia vida longa e com efeito hipnótico acentuado 2 . Deve-se considerar que apenas dois benzodiazepínicos fazem parte da lista de medicamentos para a atenção básica do município - o Diazepam e o Clonazepam e, portanto os resultados desse estudo inserem-se nesse universo. Em outros estudos realizados no Brasil observou-

\begin{tabular}{|c|c|c|c|c|c|}
\hline \multirow[t]{3}{*}{ Covariáveis } & \multicolumn{2}{|c|}{ Uso de outros psicotrópicos } & \multirow{3}{*}{$\begin{array}{c}\text { Análise bivariada } \\
\text { Valor de } p \text { * }\end{array}$} & \multicolumn{2}{|c|}{ Análise multivariada } \\
\hline & & & & RP (IC95\%) & Valor de $p$ \\
\hline & Sem uso & Com uso & & & \\
\hline \multicolumn{6}{|l|}{ Faixa etária (anos) } \\
\hline Menos de 60 & 540 & 876 & $<0,001$ & $1,63(1,42-1,86)$ & $<0,001$ \\
\hline 60 anos ou mais & 289 & 161 & & 1,00 & \\
\hline \multicolumn{6}{|l|}{ Gênero } \\
\hline Masculino & 220 & 259 & 0,443 & & \\
\hline Feminino & 609 & 778 & & & \\
\hline \multicolumn{6}{|l|}{ Inserção profissional } \\
\hline Sem inserção & 334 & 332 & 0,001 & & \\
\hline Com inserção & 150 & 229 & & & \\
\hline \multicolumn{6}{|c|}{ Medicamento prescrito } \\
\hline Diazepam & 529 & 585 & 0,001 & & \\
\hline Clonazepam & 300 & 452 & & & \\
\hline \multicolumn{6}{|c|}{ Cadastro em programas de saúde } \\
\hline Sem cadastro & 455 & 722 & $<0,001$ & $1,18(1,01-1,30)$ & 0,001 \\
\hline Cadastrado & 374 & 315 & & 1,00 & \\
\hline
\end{tabular}

IC95\%: intervalo de 95\% de confiança.

* Teste qui-quadrado de Pearson. 
Associação entre o benzodiazepínico utilizado e as covariáveis. Coronel Fabriciano, Minas Gerais, Brasil, 2006.

\begin{tabular}{|c|c|c|c|c|c|}
\hline \multirow[t]{3}{*}{ Covariáveis } & \multicolumn{2}{|c|}{ Medicamento prescrito } & \multirow{3}{*}{$\begin{array}{c}\text { Análise bivariada } \\
\text { Valor de } \mathrm{p}^{*}\end{array}$} & \multicolumn{2}{|c|}{ Análise multivariada } \\
\hline & & & & RP (IC95\%) & Valor de $p$ \\
\hline & Diazepam & Clonazepam & & & \\
\hline \multicolumn{6}{|l|}{ Faixa etária (anos) } \\
\hline Menos de 60 & 791 & 625 & $<0,001$ & 1,00 & $<0,001$ \\
\hline 60 anos ou mais & 323 & 127 & & $1,40(1,19-1,65)$ & \\
\hline \multicolumn{6}{|l|}{ Gênero } \\
\hline Feminino & 803 & 584 & 0,007 & 1,00 & 0,003 \\
\hline Masculino & 311 & 168 & & $1,23(1,07-1,41)$ & \\
\hline \multicolumn{6}{|l|}{ Inserção profissional } \\
\hline Sem inserção & 432 & 234 & & & \\
\hline Com inserção & 222 & 157 & 0,043 & & \\
\hline \multicolumn{6}{|c|}{ Cadastro em programas de saúde } \\
\hline Sem cadastro & 640 & 537 & & 1,00 & $<0,001$ \\
\hline Cadastrado & 474 & 215 & $<0,001$ & $1,35(1,19-1,55)$ & \\
\hline
\end{tabular}

IC95\%: intervalo de 95\% de confiança.

* Teste qui-quadrado de Pearson.

se também a prevalência da prescrição do Diazepam 18,19. A seleção do Diazepam em vários serviços públicos de saúde justifica-se por se tratar de medicamento pertencente à Relação Nacional de Medicamentos Essenciais-RENAME 20 , ser eficaz, seguro, de baixo custo, e de grande experiência clínica nas suas diversas aplicações 3 . As mulheres corresponderam a quase $75 \%$ dos pacientes, aspecto corroborado por vários estudos e atribuído a maior preocupação das mulheres com a própria saúde e a maior prevalência da ansiedade e depressão entre mulheres 21,22. Além desses aspectos, existem outros mais sutis que também devem ser considerados como determinantes da maior prescrição de benzodiazepínicos para mulheres. Segundo Mastroianni et al. 23 a forma estereotipada como as mulheres são apresentadas na publicidade da indústria farmacêutica interfere na abordagem clínica fazendo com que os prescritores se tornem mais suscetíveis ao diagnóstico de quadros como ansiedade e depressão no gênero feminino. É provável que, mais que uma questão de gênero, os fatores socioculturais sejam determinantes importantes desse panorama 1 .

Deve-se destacar que embora as mulheres tenham sido as maiores usuárias de benzodiazepínicos em Coronel Fabriciano, os homens apresentaram maior prevalência de utilização do Diazepam em relação ao Clonazepam. Os fatores que levam a esse resultado não são claros e devem ser melhor investigados.
O uso na população adulta correspondeu a cerca de $75 \%$ da amostra, porém o expressivo uso entre idosos confirma dados da literatura que apontam alta prevalência de utilização de benzodiazepínicos nessa faixa etária 7,10,13. A utilização do Diazepam por idosos é contraindicada e esse medicamento faz parte da lista de medicamentos de uso inadequado para idosos por estar associado ao aumento das doses relativas, dos efeitos adversos e de quedas nessa população 4,24,25,26. Embora estudos de utilização de medicamentos de base populacional ainda sejam raros no Brasil, a tendência a utilização de benzodiazepínicos por idosos parece ser uma realidade global, também evidenciada aqui, conforme apontado por Alvarenga et al. 10 em Bambuí, uma pequena cidade do interior de Minas Gerais. A prevalência de uso de benzodiazepínicos entre idosos dessa localidade foi de $21,7 \%$.

Admite-se que o consumo de benzodiazepínicos por idosos está relacionado ao fato do envelhecimento ser acompanhado pelo aparecimento de transtornos do sono, depressão e das doenças neurológicas degenerativas 26. Contudo, são quadros que merecem uma abordagem cuidadosa e uma rigorosa avaliação do benefício/ risco dessa terapia medicamentosa.

Os resultados apontaram que cerca de $40 \%$ dos usuários de benzodiazepínicos eram cadastrados nos Programas Municipais Coletivos de Saúde - Hipertensão e Diabetes, mesmo não existindo relação direta entre essas condições 
crônicas e os transtornos mentais. O uso de benzodiazepínicos em portadores de múltiplas morbidades foi descrito por Fang et al. 27 e traz preocupação quanto à possibilidade de interações medicamentosas. Embora a totalidade de medicamentos em uso no momento dessa pesquisa não tenha sido investigada, é lógico supor que pacientes pertencentes aos Programas Coletivos de Saúde utilizem outros medicamentos além do benzodiazepínico. Esses pacientes constituem um grupo de risco para interações medicamentosas. Entre os principais agravos à saúde potencialmente desencadeados pelo uso de benzodiazepínicos destaca-se o somatório do efeito depressor central do benzodiazepínico e da hipotensão ortostática comum aos anti-hipertensivos, acentuando o risco de quedas entre os pacientes particularmente entre idosos ${ }^{28}$. A relação observada entre o uso de benzodiazepínicos e o cadastro em programas de saúde é preocupante porque embora o município tenha empreendido esforços na organização dos programas, não se observaram, nesse estudo, resultados positivos em termos de menor utilização de benzodiazepínicos por esses grupos de pacientes.

O uso de benzodiazepínico mostrou maior prevalência entre pacientes que se autodeclararam não inseridos no mercado de trabalho. Outros estudos mostraram resultados semelhantes, pacientes sem uma inserção profissional apresentaram maior prevalência de doenças mentais. A falta de oportunidades de inserção no mercado de trabalho pode gerar frustrações e bloqueios sociais que acabam por desencadear um quadro de doença mental 29,30.

Dentre as questões que permeiam o uso inadequado de benzodiazepínicos o tratamento prolongado é um dos mais frequentemente relatados 3,9. Em Coronel Fabriciano, na análise bivariada a idade, o uso de Diazepam e o cadastro em programas coletivos estiveram associados ao uso prolongado de benzodiazepínicos (superior a 6 meses). Porém, na análise multivariada apenas as duas últimas variáveis se mantiveram associadas no modelo. Considera-se o uso por período superior a seis meses injustificado para a grande maioria dos quadros 2,3,5,6,9,12, com consequências danosos para os usuários, principalmente o risco de dependência 2,15,16 e gastos desnecessários para o município. $\mathrm{O}$ fato de o tratamento prolongado estar associado ao uso do Diazepam merece ser cuidadosamente avaliado. O Diazepam é um dos benzodiazepínicos mais antigos e conhecidos pela população. Estudos mostram que os prescritores tendem a manter o medicamento se o paciente já o usa há muito tempo e não se conhecem exatamente as circunstancias que levaram à indicação anterior 4,6. Essa pode ser uma das possíveis explicações para o uso prolongado associar-se a prescrição do Diazepam.

Por outro lado, a associação entre o uso prolongado e o cadastro em programas de saúde reforça a questão anteriormente colocada da incapacidade dos programas na abordagem adequada da racionalização do uso de benzodiazepínicos. Após ampla divulgação de dados e trabalhos científicos demonstrando os riscos da utilização de benzodiazepínicos por tempo prolongado, exatamente os pacientes mais assistidos pelo serviço de saúde permanecem como grandes usuários desses medicamentos.

No presente estudo buscou-se conhecer o uso do benzodiazepínico conjuntamente com outros psicotrópicos. Esta preocupação justifica-se pelo risco de complicações quando o benzodiazepínico é usado juntamente com outros medicamentos de ação central. Verificou-se que mais de $50 \%$ dos pacientes que receberam prescrição de benzodiazepínicos utilizavam pelo menos mais um psicotrópico. Embora frequentemente seja necessário usar a associação de medicamentos no manejo de doenças mentais, esse uso está relacionado ao aumento das interações medicamentosas, principalmente os efeitos aditivos com relação à depressão do sistema nervoso central 2.

A análise bivariada indicou associação estatisticamente significativa entre a idade, o tipo de benzodiazepínicos utilizado, a inserção no mercado de trabalho e o cadastro em programas de saúde com o uso de mais de um psicotrópico. A análise multivariada apontou que apenas a idade (inferior a 60 anos) e a não participação em Programas Coletivos de Saúde estão independentemente associadas à utilização concomitante de outro psicotrópico.

Deve-se considerar que os adultos são, em geral, a parcela da população economicamente ativa e responsável pelo provimento familiar. A maior prevalência do uso de múltiplos medicamentos psicotrópicos por essa parcela da população pode estar relacionada aos inúmeros fatores de estresse que se impõem sobre a população produtiva. $\mathrm{O}$ estresse no ambiente de trabalho, a má remuneração, desmotivação, jornadas longas, trabalhos de turno, ritmos intensos de trabalho e até o medo de perder o emprego podem contribuir para o adoecimento mental do paciente com quadros ansiosos, depressivos, fadiga e distúrbios do sono 31,32. Além disso, a associação observada pode indicar o uso do benzodiazepínico com o objetivo de contornar efeitos adversos, particularmente a insônia, de outras classes de psicotrópicos, como é o caso dos antidepressivos. As necessidades produtivas acabam determinando o uso de medicamentos 
para contornar efeitos adversos de um primeiro, causando outros efeitos que podem significar risco imediato ao usuário, além do comprometimento de sua qualidade de vida.

Rozemberg 11, em estudo de morbidades entre trabalhadores rurais, descreveu que os problemas laborais eram considerados pelos pacientes como a principal causa para seu adoecimento. $\mathrm{O}$ excesso de trabalho e a desvalorização externa de seu esforço foram apontados como fatores desencadeadores da doença mental.

Pacientes que não participavam dos programas municipais de saúde mostraram maior prevalência do uso de múltiplos psicofármacos. Esse achado pode estar ligado às limitações de disponibilidade dos pacientes adultos para a participação nos programas. Em geral as atividades laborais impedem a participação nesses programas.

A prescrição do Diazepam, na análise bivariada, esteve associada à idade, sexo, cadastro em programas de saúde e inserção no mercado de trabalho. Na análise multivariada apenas as três primeiras variáveis se mantiveram associadas ao desfecho.

O uso de Diazepam por pacientes idosos é considerado francamente inadequado e os protocolos de utilização reforçam os riscos inerentes desse fármaco pela população senil. No entanto, em Coronel Fabriciano, a opção pelo Diazepam esteve associada exatamente à população idosa.

Verifica-se também que o uso do Diazepam associou-se ao cadastro em programas de saúde, reforçando a preocupação com as comorbidades que, em geral, implicam no uso de múltiplos medicamentos, potencializando o risco das interações medicamentosas.

Deve-se considerar que o estudo tem limitações decorrentes do método utilizado que não assegura que o medicamento dispensado foi efetivamente utilizado, da ausência de informação sobre inserção no mercado de trabalho de cerca de $50 \%$ da amostra e, particularmente, do fato de o cadastro em programas de saúde não signi- ficar necessariamente a participação efetiva do paciente nesse programa. Além disso, ressalta-se que o total de pacientes inseridos nos programas coletivos de saúde pode ter sido subestimado, uma vez que esse campo do cadastrado do paciente no sistema informatizado foi excluído em 2005. É importante destacar, ainda, que o Brasil tem no Formulário Terapêutico Nacional (FTN) 33, uma fonte oficial de indicação de benzodiazepínicos. De acordo com o FTN, tanto para o tratamento da ansiedade, como para a insônia, o uso de benzodiazepínicos deveria ocorrer por curto período de tempo (7-10 dias). Outros protocolos, entretanto, adotam intervalos maiores. Para fins de análise nesse artigo optou-se por utilizar uma posição mais conservadora, com tempo de uso superior a seis meses, como previsto em outras referências 15,16 .

Contudo, acredita-se que o tamanho da amostra, a coleta de dados realizada por uma única pesquisadora - farmacêutica do município - e os protocolos de busca do registro informatizado e de confirmação dos dados coletados reforçam a consistência dos resultados encontrados.

O panorama de utilização de benzodiazepínicos em Coronel Fabriciano, cidade localizada no interior de Minas Gerais, apontando o uso prolongado de benzodiazepínicos, o uso extensivo de Diazepam por idosos e por pacientes cadastrados nos Programas Coletivos de Saúde e o uso concomitante de psicotrópicos juntamente com o benzodiazepínico são aspectos demonstrados no presente estudo. Os resultados evidenciam a amplitude do problema e devem ser considerados no planejamento de intervenções para a racionalização da utilização desses medicamentos no município, particularmente na organização dos programas de saúde. O espaço do programa mostra-se como uma oportunidade para identificação dos pacientes com transtornos ansiosos e do sono, promovendo terapias de apoio, evitando-se o uso do benzodiazepínico. 


\section{Resumo}

O uso inadequado de benzodiazepínicos é observado em diversos países. Este estudo transversal avaliou os fatores associados à prescrição de benzodiazepínicos para os usuários do Serviço Municipal de Saúde de Coronel Fabriciano, Minas Gerais, Brasil. A coleta de dados foi feita a partir da notificação das receitas ( $n=1.866)$, entre setembro e outubro de 2006. Análises bivariada e multivariada, utilizando regressão de Poisson, foram executadas. O uso prolongado de benzodiazepínicos foi associado independentemente com o tipo de benzodiazepínico prescrito e cadastro em programas de saúde. O uso concomitante de outros psicofármacos foi associado independentemente com a idade e cadastro nos programas de saúde. O tipo de benzodiazepínico utilizado (Diazepam ou Clonazepam) esteve independentemente associado com a idade e sexo dos pacientes, bem como, com a participação em programas de saúde. Os fatores associados à prescrição de benzodiazepínicos evidenciam a amplitude do problema e devem ser considerados no planejamento de intervenções para a racionalização da utilização desses medicamentos no município, particularmente na organização dos programas de saúde.

Benzodiazepinas; Psicotrópicos; Farmacoepidemiologia

\section{Colaboradores}

M. H. N. G. Abreu colaborou na análise, interpretação dos dados, redação e revisão crítica do conteúdo intelectual. S. M. S. Magalhães participou da concepção do projeto e interpretação dos dados, redação e revisão crítica relevante do conteúdo intelectual e; foi orientadora do trabalho. E. Perini contribuiu com a concepção do projeto, interpretação dos dados e revisão crítica relevante do conteúdo intelectual. K. F. Firmino participou da concepção do projeto, coleta dos dados, análise e interpretação dos dados, redação do artigo e da sua revisão crítica.

\section{Referências}

1. Silveira ML. O nervo cala, o nervo fala: a linguagem da doença. Rio de Janeiro: Editora Fiocruz; 2000. (Coleçäo Antropologia \& Saúde).

2. Sweetman SC. Martindale: the complete drug reference. 34th Ed. London: Pharmaceutical Press; 2005.

3. Galleguillos T, Risco L, Garay JL, González M, Vogel M. Tendencia del uso de benzodiazepinas en una muestra de consultantes en atención primaria. Rev Méd Chile 2003; 131:535-40.

4. Cook JM, Marshall R, Masci C, Coyne JC. Physicians' perspectives on prescribing benzodiazepines for older adults: a qualitative study. J Gen Intern Med 2007; 22:303-7.
5. Dièye AM, Sylla M, Ndiaye M, Yoro Sy G, Faye B. Benzodiazepines prescription in Dakar: a study about prescribing habits and knowledge in general practitioners, neurologists and psychiatrists. Fundam Clin Pharmacol 2006; 20:235-8.

6. Auchewski L, Andreatini R, Galduróz JCF, Lacerda RB. Avaliação da orientação médica sobre os efeitos colaterais de benzodiazepínicos. Rev Bras Psiquiatr 2004; 26:24-31.

7. Rancourt C, Moisan J, Baillargeon L, Verreault R, Laurin D, Grégoire J. Potentially inappropriate prescriptions for older patients in long-term care. BMC Geriatr 2004; 4:1-9. 
8. Valenstein M, Taylor KK, Austin K, Kales HC, McCarthy JF, Blow FC. Benzodiazepine use among depressed patients treated in mental health settings. Am J Psychiatry 2004; 161:654-61.

9. Poyares D, Ohayon MM, Guilleminault C, Tufik S. Chronic benzodiazepine usage and withdrawal in insomnia patients. J Psychiatr Res 2004; 38:327-34.

10. Alvarenga JM, Loyola Filho AG, Firmo JOA, LimaCosta MF, Uchôa E. Prevalence and sociodemographic characteristics associated with benzodiazepines use among community dwelling older adults: the Bambuí Health and aging study (BHAS). Rev Bras Psiquiatr 2008; 30:7-11.

11. Rozemberg B. O consumo de calmantes e o "problema de nervos" entre lavradores. Rev Saúde Pública 1994; 28:300-8.

12. Laranjeira R, Castro LAPG. Dependência de benzodiazepínicos. http://www.uniad.org.br (acessado em 08/Abr/2006)

13. Coelho Filho JM, Marcopito LF, Castelo A. Perfil de utilização de medicamentos por idosos em área urbana do Nordeste do Brasil. Rev Saúde Pública 2004; 38:557-64.

14. Ministério da Saúde. Portaria no ${ }^{\circ} 344$ regulamenta o uso de medicamentos sujeitos a controle especial. http://www.e-legis.anvisa.gov.br (acessado em 03/Nov/2007).

15. Royal Australian College of General Practitioners. Guidelines for benzodiazepine. http://www.racgp. org.au/guidelines (acessado em 16/Ago/2007).

16. Ford C, Kay R, Barjolin JC. Guidance on prescribing benzodiazepines to drugs users in primary care: substance misuse management in general practice. http://www.smmgp.org.uk (acessado em 20/Ago/2007).

17. Vigo A. Modelando desfechos comuns: viés e precisão. Cad Saúde Pública 2006; 21:2496-7.

18. Mendonça RT, Carvalho ACD. O papel de mulheres idosas consumidoras de calmantes alopáticos na popularização do uso destes medicamentos. Rev Latinoam Enferm 2005; 13:1207-12.

19. Noto AR, Carlini EA, Mastroianni PC, Alves AC, Galduróz JCF, Kuroiwa W, et al. Análise de prescrições e dispensação de medicamentos em duas cidades no estado de São Paulo, Brazil. Rev Bras Psiquiatr 2002; 24:68-73.

20. Gerência Técnica de Assistência Farmacêutica, Departamento de Atenção Básica, Secretaria de Políticas de Saúde, Ministério da Saúde. Relação nacional de medicamentos essenciais - RENAME. 4a Ed. Brasília: Ministério da Saúde; 2007.

21. Rasu RS, Shenolikar RA, Nahata MC, Balkrishnan R. Physician and patient factors associated with the prescribing of medication for sleep difficulties that are associated with high abuse potencial or are expensive: an analysis of data from the National Ambulatory Medical Care Survey for 1996-2001. Clin Ther 2005; 27:1970-9.
22. Andrade L, Walters EE, Gentil V, Laurenti R. Prevalence of ICD-10 mental disorders in a catchment area in the city of São Paulo, Brazil. Soc Psychiatry Psychiatr Epidemiol 2002; 37:316-25.

23. Mastroianni P, Galduróz JCF, Carlini EA. Influence of the legislation on the advertisement of psychoactive medications in Brazil. Rev Bras Psiquiatr 2003; 25:146-55.

24. Passaro A, Volpato S, Romagnoni F, Manzoli N, Zuliani G, Fellin R. Benzodiazepines with different half-life and falling in a hospitalized population: the GIFA study. J Clin Epidemiol 2000; 53:1222-9.

25. Fick DM, Cooper JW, Wade WE, Waller JL, Maclean JR, Beers MH. Updating the Beers criteria for potentially inappropriate medication use in older adults: results of a US consensus panel of experts. Arch Intern Med 2003; 163:2716-24.

26. Chaimowicz F, Ferreira TJXM, Miguel DFA. Use of psychoactive drugs and related falls among older people living in a community in Brazil. Rev Saúde Pública 2000; 34:631-5.

27. Fang SY, Chen CY, Chang IS, Wu ECH, Chang CM, Lin KM. Predictors of the incidence and discontinuation of long-term use of benzodiazepines: a population-based study. Drug Alcohol Depend 2009; 104:140-6.

28. Glass LKL, Herrmann N, Sproule BA, Busto UE. Sedative hypnotics in older people with insomnia: meta-analysis of risks and benefits. BMJ 2005; 331:1169-72.

29. Andrews G, Henderson S, Hall W. Prevalence, comorbidity, disability and service utilization. Br J Psychiatr 2001; 178:145-53.

30. Ludermir AB, Melo Filho DA. Condições de vida e estrutura ocupacional associada a transtornos mentais comuns. Rev Saúde Pública 2002; 36: 213-21.

31. World Health Organization. Improving access and use of psychotropic medicines. Mental health policy and service guidance package. Geneva: World Health Organization; 2005.

32. Ministério da Saúde/Organização Pan-Americana da Saúde. Doenças relacionadas ao trabalho: manual de procedimentos para os serviços de saúde. Brasília: Ministério da Saúde; 2001.

33. Ministério da Saúde. Formulário terapêutico nacional 2008: RENAME 2006. Brasília: Ministério da Saúde; 2008.

Recebido em 19/Abr/2010

Versão final reapresentada em 24/Ago/2010

Aprovado em 26/Abr/2011 